\title{
Order information in short-term memory and time estimation
}

\author{
CLAUDETTE FORTIN and NATHALIE MASSÉ \\ Université Laval, Québec, Québec, Canada
}

\begin{abstract}
In previous experiments, the amount of interference between time production and visual or memory search tasks was shown not to be related to the level of difficulty of the search task per se, but instead to the amount of processing in short-term memory required in the search task. The first experiment of the present study verified whether the amount of interference between time production and a short-term memory task may be related to the level of difficulty of the short-term memory task. Two versions of a memory task, with and without processing of order information, were combined with a temporal interval production task in a concurrent processing condition. As is shown in a control reaction time task, processing order information increased the level of difficulty of the memory search task. In the concurrent processing condition, the interference between short-term memory processing and time production was stronger when the level of difficulty of the short-term memory search task was increased by requiring that order information be processed. The results of Experiment 2 showed that the amount of interference between a similar short-term memory task and time production seems not to be related to the amount of order information that must be maintained during the time production task. This dissociation between the effects of processing and the maintenance of order information is compatible with a similar dissociation, observed in previous experiments, between the effects of processing and those of maintaining item information in short-term memory on concurrent time production.
\end{abstract}

A current view, in time estimation research, holds that short-term or working memory is involved in performing the time estimation tasks used in most time research paradigms (Zakay, 1990). For example, a well-known psychophysical model of time estimation assumes that, to reproduce a brief time interval (e.g., $2 \mathrm{sec}$ ), temporal information would be accumulated that could then be stored in a short-term memory store during the temporal reproduction. This information would be compared with a criterion amount of temporal information, corresponding to the to-be-reproduced interval, stored in long-term memory (Church, 1984; Gibbon, Church, \& Meck, 1984). Some recent research with human subjects suggests that shortterm memory would also be involved in the comparison of brief time intervals (Wearden \& Ferrara, 1993).

Fortin and Rousseau (1987) combined a short-termmemory search task with time interval production. In this experiment, the subject memorized a set of digits and then produced a subjective 2-sec interval. During the temporal production, a probe digit was presented. At the end of the subjective 2-sec interval, the subject was asked to press one of two response buttons: One was used for answering

This research was supported by a grant from the Natural Science and Engineering Research Council of Canada to the first author. We would like to thank Y. Lacouture for helpful suggestions regarding this research and M. Poirier for useful comments on an earlier version of this manuscript. Correspondence should be addressed to C. Fortin, École de Psychologie, Université Laval, Québec, QC G1K 7P4, Canada (e-mail: claudette.fortin@psy.ulaval.ca). that the probe was present in the memory set, whereas the other was to be pressed to indicate that the probe was absent. Thus, the subject had to perform the memory search task and to estimate time concurrently. Complexity in memory search was manipulated by varying the number of items in the memory set $(n=1-6)$. In that dual-task situation, the mean produced temporal interval was lengthened proportionally to the number of items in the memory set - that is, to the complexity of the nontemporal task.

In a series of further experiments, Fortin, Rousseau, Bourque, and Kirouac (1993) used the same concurrent search/time-production paradigm with four different search tasks. In each of these memory or visual search tasks, the complexity of the search, defined in resource theory as the size of the visual or memory set (Navon, 1984), was varied. The level of difficulty, defined as the amount of resources necessary to maintain a given speed or accuracy (Navon, 1984), was approximately the same, as estimated in reaction time (RT) conditions: The four tasks could all be performed at an average rate of about $40 \mathrm{msec}$ per item. However, the tasks differed in their short-term memory processing requirements. The results showed a clear dissociation between RT and time production data as the complexity-that is, the size of the set-was varied in the search task. Whereas increasing complexity in a short-term memory search task lengthened temporal productions proportionally to memory set size, a similar increase of complexity in the visual search task had no effect on concurrent temporal productions. These tasks being of comparable levels of difficulty, as measured by RT, the amount of interference with time production was shown 
not to be related to their level of difficulty, but instead to their short-term memory processing requirements.

These results were interpreted within psychophysical models of time estimation: A time interval production requires the accumulation of a number of pulses emitted from an internal source until a criterion number of pulses, corresponding to the target duration, is reached (Church, 1984; Gibbon et al., 1984). In some timing models, the accumulation process is under attentional control by a switch (Meck, 1984) or a gate (Rousseau, Picard, \& Pitre, 1984; Zakay \& Block, 1997a) that enables the accumulation while in an "on" state. Concurrent nontemporal processing would put the gate in an "off" state and would temporarily interrupt the accumulation process. This would increase the time required to reach the criterion number of pulses, thus lengthening the produced time interval. Inasmuch as increasing set size in concurrent visual search tasks had no effect on temporal production duration, whereas a proportional lengthening of produced intervals with set size in concurrent short-term memory tasks was observed, Fortin et al. (1993) suggested that "the attentional gate enabling temporal information accumulation or the accumulation process itself, is under short-term memory control" (p. 547).

Because concurrent tasks of the same level of difficulty showed different patterns of interference with time production in these experiments, the results were also difficult to account for with attentional models of time estimation without considering some contribution of shortterm memory to time estimation. Within that framework, a cognitive timer requires attention to count the subjective time units, and the length of an estimated interval will be related to the amount of attentional resources allocated to the timer. Thus, during a to-be-estimated inter$\mathrm{val}$, if a nontemporal task also requiring attention is executed, the estimated interval should be shortened, because less attention will be devoted to the timer. Results in time estimation research have often supported this attentional approach, showing a negative relationship between nontemporal task difficulty and perceived duration in a prospective time estimation paradigm - that is, one in which the subjects know in advance that they have to estimate time (see, e.g., Block, 1992; Hicks, Miller, Gaes, \& Bierman, 1977; Hicks, Miller, \& Kinsbourne, 1976; Macar, Grondin, \& Casini, 1994; McClain, 1983; Thomas \& Weaver, 1975; Vroon, 1970; Zakay, 1993; Zakay, Nitzan, \& Glickson, 1983).

In a temporal production paradigm, if a nontemporal task requiring attention is executed during time production, less attention will be allocated to the counting of time units, so that more real time will be needed to accumulate the amount of temporal information corresponding to the target interval. In this situation, a lengthening of produced time intervais as a function of increasing concurrent nontemporal task difficulty is expected (Zakay $\&$ Block, 1997b). This approach would have predicted that, in Fortin et al.'s (1993) study, search tasks of the same level of difficulty should have the same effect on concurrent time production, which was not actually observed. In fact, time intervals lengthened with the amount of processing in a concurrent task involving short-term processing but did not vary with increasing the amount of processing in concurrent visual search tasks that did not require short-term or working memory processing. As with timing models integrating attention (Meck, 1984; Rousseau et al., 1984; Zakay \& Block, 1997a), some shortterm memory control should be integrated to attentional models of time intervals estimation to account for Fortin et al.'s results.

However, the fact that various search tasks of the same difficulty show different levels of interference with time production does not imply that varying the level of difficulty within a nontemporal task that interferes with temporal production would not make the temporal production vary accordingly. More precisely, the amount of interference from a short-term memory task on concurrent time estimation could be specifically related to the level of difficulty of the short-term memory task. This would be concordant with the numerous observations relating attention and time estimation and, generally, with time estimation models that consider the role of attention in temporal estimation.

In Experiment 1 of the present study, the effect of varying the ievel of difficulty of short-term memory processing on concurrent time production was examined. In addition to performing a standard memory search task combined with time production, the subjects were asked to process order information in short-term memory. Processing order information in a memory search task has been shown to increase the difficulty of the search task. For example, in a variant of Sternberg's (1966) classical paradigm, subjects were asked to scan the memory set for location. In this condition, the slope of RTs as a function of memory set size was steeper than the slope observed when subjects only had to scan for presence of the probe in the set $(85 \mathrm{msec}$ per item vs. $50 \mathrm{msec}$ per item; Chase, 1977; Sternberg, 1967, 1969). In Experiment 2, the memory search with order information task was slightly modified so that processing of order could be postponed until the end of the time interval production; in this experiment, order information would only have to be maintained during time production.

\section{EXPERIMENT 1}

The purpose of the first experiment was to verify whether increasing the level of difficulty of a short-term memory search task, by requiring that order information be aiso processed, would increase the amount of interference between this search task and concurrent time production. The interference was tested in a concurrent processing (CP) condition, in which two conditions were compared: memory search + time production (MP) and memory search + processing of order + time production 
(MOP). An RT task was used to determine whether reporting the location of the probe increased the difficulty of the search task. Two RT conditions were included: memory search only $(\mathrm{M})$ and memory search + processing of order (MO). In the RT conditions, the dependent variable was RT, whereas in the CP conditions, the dependent variable was the duration of temporal intervals produced by the subjects.

Method
Subjects
Forty subjects, 20 male and 20 female, between 19 and 42 years
old (mean age $=23.4)$ took part in this experiment. They were
mostly undergraduate or graduate students at Laval University. The
subjects in the RT conditions were paid $\$ 10$ for their participation
in the three experimental sessions, and the subjects in the CP con-
ditions were paid $\$ 20$ for the three practice sessions plus two ex-
perimental sessions. They were all naive regarding the hypotheses
of the experiment.

\section{Apparatus and Stimuli}

Stimulus and feedback presentations as well as data collection were controlled by a PC-compatible computer running MEL software. The visual stimuli were displayed on an IBM VGA color monitor with a $20 \times 27 \mathrm{~cm}$ screen. Responses (RTs and temporal productions) were provided by pressing one of three keys on the numerical keyboard of the computer. The subjects were tested individually in a sound-attenuated test chamber, where they were seated in front of the screen at a distance of about $60 \mathrm{~cm}$.

The set of items used in the experiment comprised the 20 consonants of the alphabet. The memory set size $(n=2,4$, or 6 different consonants) and the probe consonant $(n=1)$ were selected randomly and varied from trial to trial. The consonants were presented in white on a black background and subtended a visual angle of $0.2^{\circ}$ in height and $0.4^{\circ}$ in width.

\section{Procedure}

Twenty subjects were randomly assigned to the RT conditions, in which each subject was successively tested in the $M$ and the MO conditions, in two separate sessions. The order of testing in the $M$ and MO conditions was counterbalanced. Twenty other subjects were tested in the CP conditions, which included two successive experimental sessions in the MP and MOP conditions, counterbalanced across subjects. In the RT conditions, the subjects performed the memory search task with the instruction to respond as quickly as possible to the presence or absence of the probe, while minimizing errors. In the CP conditions, the subjects first practiced to produce a given time interval during practice sessions, with feedback informing the subjects whether their production was too long, too short, or correct within a $10 \%$ window centered on the 2 -sec target interval. The objective of these sessions was to stabilize the temporal production performance. Then, the subjects were tested in experimental sessions, in which they were instructed to produce as closely as possible the target interval that they had practiced previously, while simultaneously executing the memory search task.

Reaction times conditions. Each of the two experimental sessions included four blocks of 36 trials, with a 30 -sec rest between blocks. Sessions lasted about $30 \mathrm{~min}$. An experimental trial began with the presentation of a fixation point. The subject was instructed to put three fingers of the same hand on the three adjacent keys " 1 ", " 2 ," and " 3 " of the numerical keyboard. When ready to begin the trial, the subject pressed the " 2 " on the keyboard. The fixation point reappeared for $500 \mathrm{msec}$ and, after this delay, was followed by the presentation of the memory set. Each item of the memory set was presented for $1 \mathrm{sec}$, with no delay between items. After the memory set presentation, the fixation point reappeared and remained present until the subject pressed the " 2 " key once more. Five hundred msec later, a probe consonant appeared and remained present until the subject responded to the search. In the $\mathrm{M}$ condition, if the probe belonged to the memory set (positive trial), the " 3 " key was to be pressed, and if it did not belong to the memory set (negative trial), the " 1 " was to be used. In the M condition, a neutral stimulus (\#) was presented below the probe. In the MO condition, a digit was presented below the probe; a positive response was required when the probe was present in the memory set and the digit indicated the correct location of the probe in the memory set. Schematic illustrations of experimental trials in the $\mathrm{M}$ and $\mathrm{MO}$ conditions are presented in Figures $1 \mathrm{a}$ and $1 \mathrm{~b}$, respectively. In both conditions, a feedback was then presented, informing the subject whether the response was correct or not. The feedback was followed by a fixation point indicating the beginning of the next trial. The position of the probe was selected randomly. Time and accuracy of the responses were collected.

Concurrent processing conditions. In the $\mathrm{CP}$ conditions, there were five sessions: three practice sessions of temporal production alone and two experimental sessions in which the memory search task was combined with temporal production. The practice sessions included four 48-trial blocks, with feedback on the temporal performance, and one block without feedback. The experimental sessions were composed of one 48-trial block of practice of temporal production alone, with feedback on the temporal performance as in the practice sessions, and then of four 36-experimental-trial blocks in which temporal production was combined with memory search. There was always a 30 -sec break between blocks. Sessions lasted between 30 and $45 \mathrm{~min}$.

In the practice sessions, examples of the 2-sec target interval were presented to the subjects without referring to conventional units of time in the first practice session. Then the subject was asked to produce himself/herself the target interval by pressing a key two times in succession. A trial started with the presentation of a fixation point. When ready, the subject started the interval by pressing the " 2 " key on the keyboard. The fixation point remained present for $500 \mathrm{msec}$ and was then replaced by an arrow indicating which key (" 1 " on the left or " 3 " on the right) to press when the subjective 2-sec interval had elapsed. This procedure aimed at familiarizing the subject with the dual response that would be required in the experimental sessions. In the first four blocks of the practice sessions, a visual feedback for the temporal production task was provided on each trial, indicating whether the temporal production was too short, too long, or correct within a 200 -msec window centered on the 2-sec standard. In the fifth and last block of the practice sessions, no feedback was provided, to train the subject to produce without feedback.

In the experimental sessions, a first block of temporal production alone was followed by four experimental trial blocks. In these blocks, an experimental trial began with the presentation of a fixation point. The subject was instructed to put three fingers of the same hand on the three adjacent keys " 1, ," 2 ," and " 3 " of the numerical keyboard. When ready to begin the trial, the subject pressed the "2" key on the keyboard. The fixation point reappeared for $500 \mathrm{msec}$ and, after this delay, was followed by the presentation of the memory set. Each item of the memory set was presented for $1 \mathrm{sec}$, with no delay between items. After the memory set presentation, the fixation point reappeared and remained present until the subject began the time interval by pressing the "2" key. Five hundred msec later, a probe appeared and remained present until the subject ended the interval by pressing the " 3 " key, to provide a positive response to the search task, or the " 1 " key, to provide a negative response. In the MP condition, the probe with neutral stimulus was presented as in the $M$ condition, and the response was positive 
a)
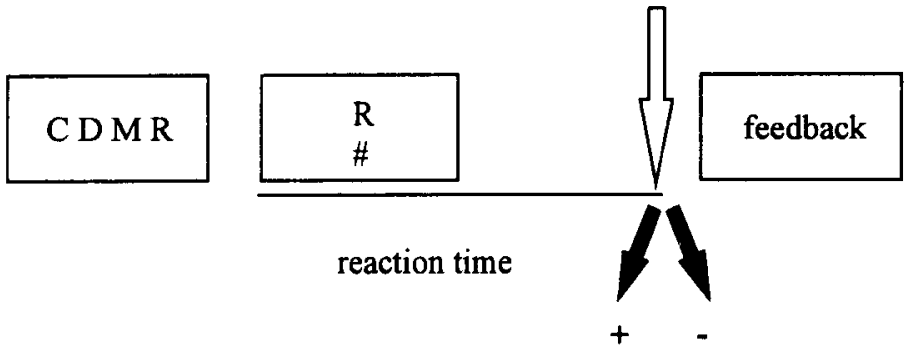

b)
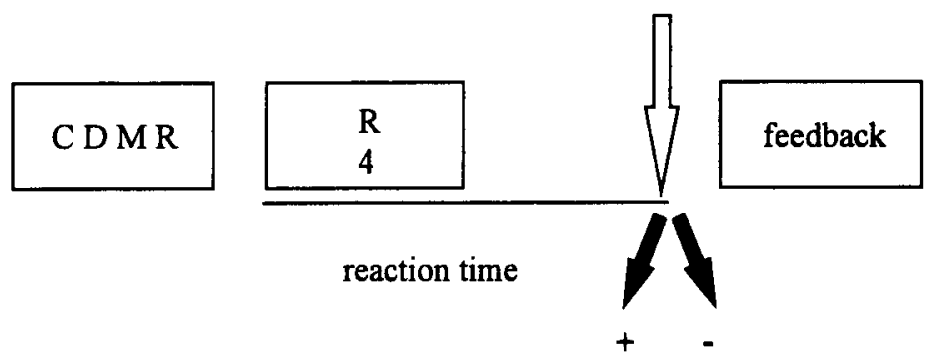

c)

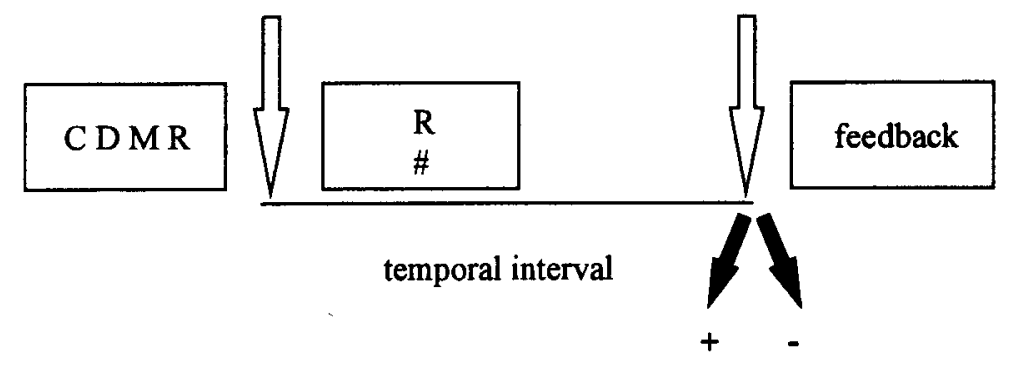

d)

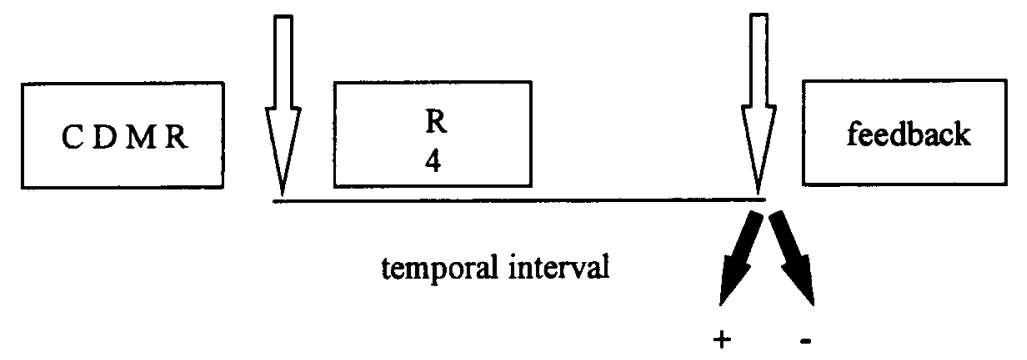

Figure 1. Experiment 1. (a) Schematic description of experimental trials in the memory search only condition. A set of consonants is presented. Then a probe and a neutral stimulus (\#) are presented. The subject indicates as quickly as possible whether the probe belonged (positive response) or did not belong (negative response) to the memory set. (b) In the memory search + processing of order condition, a digit is presented below the probe. The response is positive if the probe was present in the memory set and the digit indicates its correct location. Otherwise, the response is negative. (c) In the memory search + time processing condition, a probe and a neutral stimulus are presented during the temporal interval production. The subject ends the temporal interval production by pressing one of two buttons, depending on the presence or absence of the probe in the memory set. (d) In the memory search + processing of order + time production condition, the interval is ended by pressing one button if the response is positive (probe present and correct location), another button if the response is negative.

if the probe belonged to the memory set, as is illustrated in Figure 1c. In the MOP condition, the probe was presented with a digit, and the response was positive if the probe was present and the digit indicated the correct location of the probe in the set (Figure Id). After the temporal interval, feedback was provided, indicating whether the response to the search was correct or not. This was followed by a fixation point indicating the beginning of the next trial.

In the M and MP conditions, the memory set size was varied, the probe could be present or absent of the memory set, and these factors were balanced within blocks. In the MO and MOP conditions, 


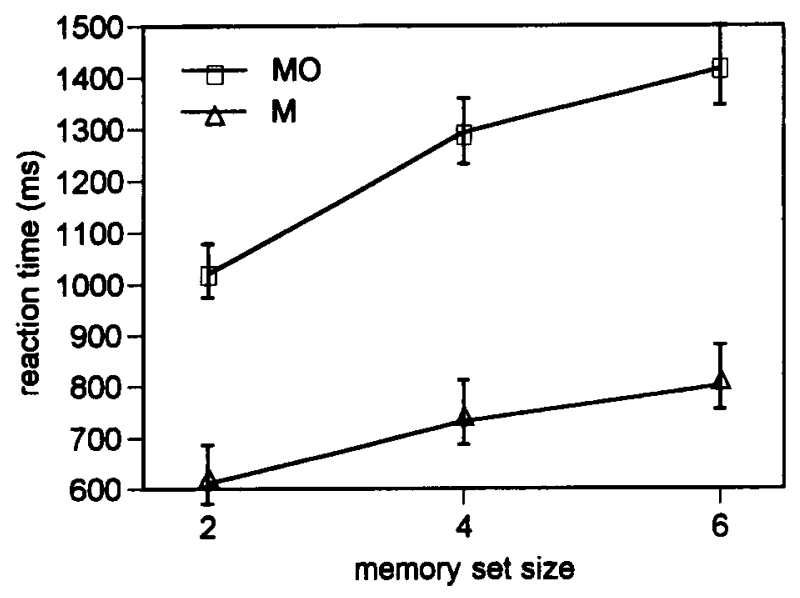

Figure 2. Experiment 1. Mean reaction time (and standard error of the means) as a function of memory set size in the memory search + processing of order (MO) and memory search only (M) conditions.

there were three types of trials - probe present and correct location (positive-positive trial), probe present and wrong location (positive-negative), and probe absent (negative). The memory set size and type of trials were balanced within blocks. The RT was measured in six treatment combinations, defined by two within-groups factors: processing of order (order and no-order in the $\mathrm{MO}$ and $\mathrm{M}$ conditions, respectively) and memory set size $(n=2,4$, or 6 , three levels). The temporal intervals were measured in the corresponding conditions defined by the MOP and MP conditions and the three memory set sizes.

RTs, in the RT conditions, and temporal intervals, in the CP conditions, were measured to the nearest millisecond. Trials on which an error was made in the memory search task were eliminated from the analyses. After removal of these data, the means and standard deviations of RTs and temporal intervals were computed for each subject, and RTs more than three standard deviations from the means were discarded.

In each condition-M, MO, MP, and MOP-2,880 experimental trials were executed. In the $\mathrm{M}$ and $\mathrm{MO}$ conditions, respectively, $7.7 \%$ and $11.7 \%$ of the data were removed because of errors, and $2.0 \%$ and $1.3 \%$ outliers were eliminated. In the MP and MOP conditions, $5.4 \%$ and $8.0 \%$ of errors and $1.5 \%$ and $1.4 \%$ outliers were eliminated. The data from the experimental sessions were averaged to get, for each subject, a mean RT at each memory set size in the $\mathrm{M}$ and $\mathrm{MO}$ conditions and a mean temporal production at each memory set size in the MP and MOP conditions. Repeated measures analyses of variance (ANOVAs) were carried out on mean RTs in the RT condition and on mean temporal intervals in the CP condition. ANOVAs were also performed on mean percent error rates. When necessary, the Greenhouse-Geisser adjustment factor was applied to degrees of freedom (see Kirk, 1995). The level of statistical significance was set at $p<.05$.

\section{Results and Discussion}

The mean RTs as a function of memory set size, in the $\mathrm{M}$ and MO conditions, are shown in Figure 2. Mean RTs increased significantly with memory set size $[F(2,38)=$ $73.82, p<.0001]$ and were longer in the MO than in the $M$ condition $[F(1,19)=146.69, p<.0001]$. The interaction between these factors was also significant $[F(2,38)=$ $22.63, p<.0001]$. The slopes and intercepts of mean RTs as a function of memory set size are 47 and $543 \mathrm{msec}$, respectively, in the $\mathrm{M}$ condition and 100 and $851 \mathrm{msec}$ in the MO condition.

A popular stage model of the internal events occurring during a memory search task assumes that the following operations contribute to increased RT in this task: stimulus encoding, comparison of the probe with set items, response selection, and response execution (Townsend \& Ashby, 1983). The duration of the comparison process is usually assumed to be set-size dependent, whereas stimulus encoding, response selection, and response execution are thought not to be affected by set size. In the MO condition, the intercept is about $300 \mathrm{msec}$ higher than in the $\mathrm{M}$ condition. This may be due to operations occurring during the stimulus encoding and the response selection stages-for example, to encode the digit in the MO condition and, in the response selection stage, to decide whether the digit corresponds to the probe's position. The steeper slope, in the MO condition, would be due to the comparison process, the duration of which is lengthened proportionally to set size. According to resource theory, this increase in RT slope as a function of set size indicates an increase in difficulty level, implying that the amount of resources necessary to maintain a given speed in the search task is higher in the MO condition than in the M condition (Navon, 1984).

In the $\mathrm{CP}$ conditions, the results are similar, as can be seen in Figure 3. Mean temporal intervals lengthened with memory set size $[F(2,38)=16.14, p<.0003]$ and were longer in the MOP condition $[F(1,19)=7.18, p<.02]$. The interaction between these factors was significant $[F(2,38)=7.40, p<.008]$. Again, the intercept and slopes of the mean temporal intervals function were higher with processing of order: 2,058 and $21 \mathrm{msec}$ in the MP condition and 2,092 and $57 \mathrm{msec}$ in the MOP condition.

In the MP condition, the proportional lengthening of mean temporal productions with increasing set size in

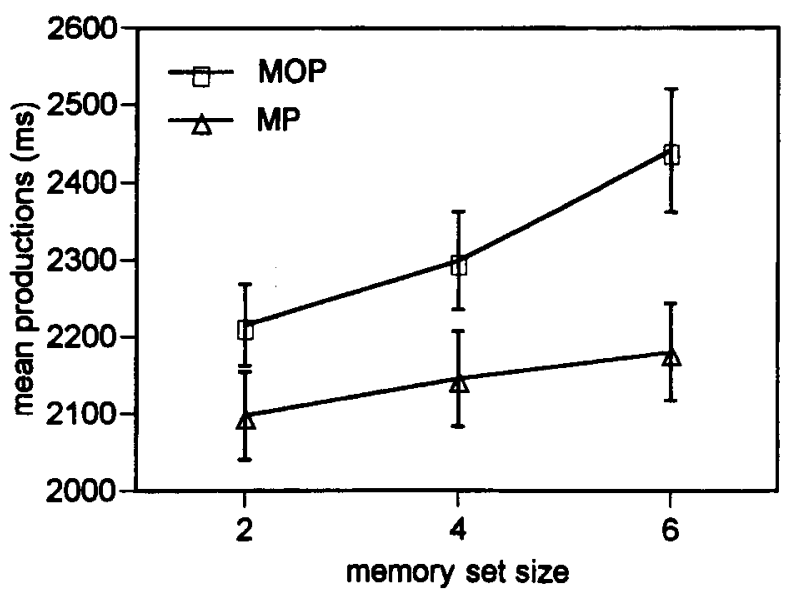

Figure 3. Experiment 1. Mean temporal production (and standard error of the means) as a function of memory set size in the memory search + processing of order + time production (MOP) and memory search + time processing (MP) conditions. 
Table 1

Mean Percent Error Rates for Each Set Size in the Reaction Time and Concurrent Processing Conditions in Experiment 1

\begin{tabular}{lrcc} 
& \multicolumn{3}{c}{ Memory Set Size } \\
\cline { 2 - 4 } Condition & 2 & 4 & 6 \\
\hline Reaction time & & & \\
M & 2.6 & 6.4 & 14.3 \\
$\quad$ MO & 4.7 & 12.1 & 18.2 \\
$\quad \begin{array}{l}\text { Concurrent processing } \\
\text { MP }\end{array}$ & 1.1 & 5.1 & 10.1 \\
MOP & 3.5 & 3.2 & 17.3 \\
\hline
\end{tabular}

Note--M, memory search; MO, memory search + processing of order; MP, memory search + time production; MOP, memory search + processing of order + time production.

memory search is replicated. In the MOP condition, the slope is much steeper than in the MP condition, and this difference was significant, as is confirmed by the significant interaction. This result shows that the amount of interference between time production and short-term memory processing is related to the level of difficulty of the search task. More precisely, the lengthening of temporal productions is proportional to the duration of the comparison process, when this duration is determined by the level of difficulty of the memory search task.

These results support models of time estimation that include attention, such as timing models integrating attentional control (Meck, 1984; Rousseau et al., 1984; Zakay \& Block, 1997a). These models assume that the accumulation process is under the control of a switch or of a gate that may enable or prevent the accumulation of temporal information emitted from an internal source. The duration of interruption in the accumulation process, during time production, could be related to the speed of probe comparison with memorized items in the search task.

The intercept is also slightly higher in the MOP than in the $\mathrm{M}$ condition ( $34 \mathrm{msec}$ ), suggesting that nontemporal operations other than comparison also interfered with time estimation. Thus, the decision process related to the probe's position may have added to the temporal interval duration. Although encoding the digit, in the MOP condition, could also have lengthened the interval duration, it was shown clearly in a similar concurrent processing situation that increasing the number of items to process in a visual search task did not interfere with concurrent time production (Fortin et al., 1993).

Mean percent error rates for each combination of set size and order condition are shown in Table 1. Although these results must be interpreted with caution because the sample size is small, ANOVAs were run on mean percent error rates. Error rates increased with set size in the RT conditions $[F(2,38)=71.19, p<.0001]$ and were higher in the MO than in the $\mathrm{M}$ condition $[F(1,19)=$ $10.60, p<.004]$. The interaction between set size and order condition was also significant $[F(2,38)=11.43, p<$ $.0005]$. In the $\mathrm{CP}$ conditions, error rates increased with set size $[F(2,38)=56.17, p<.0001]$ and were higher in the MOP than in the MP condition $[F(1,19)=24.83, p<$
$.0001]$. The interaction between these two factors was not significant $[F(2,38)=1.79, p<.18]$. Generally, errors tend to increase with set size and to be longer when processing of order is required. These results are similar to those observed with RTs and temporal intervals, showing that the longer RTs in the MO and MOP conditions were not due to a higher priority allocated to accuracy in these conditions.

Overall, the results of Experiment 1 show that increasing the level of difficulty of a short-term memory search task by requiring that order information be processed increases the amount of interference between this search task and concurrent time production. This interference seems to be induced mainly by the lengthening of the comparison process in the search task, although some other operations related to processing of order, independent of set size, might also interfere with time production. These results support models incorporating attentional control in time estimation (Meck, 1984; Rousseau et al., 1984; Zakay \& Block, 1997a) and generally are consistent with attentional models of time estimation (see, e.g., Thomas \& Weaver, 1975). It must be noted that this relation between difficulty and interference with time production was not observed when difficulty was constant across various search tasks that involved different amounts of shortterm memory processing (Fortin et al., 1993). However, within a short-term memory task, the level of difficulty appeared to be related to the amount of interference with concurrent time production in Experiment 1.

\section{EXPERIMENT 2}

In the MOP condition of Experiment 1, subjects had to process item and order information during the time interval production. At the end of the subjective target interval, the response was positive if the probe letter was present and located in the position indicated by the digit. Therefore, during the time interval, two decisions were made: whether the probe was present in the memory set (processing of item information) and, when present, whether it was in the position indicated by the digit (processing of order information). This MOP condition was compared with an MP condition, and the two conditions were shown to interact with set size.

In one condition of Experiment 2, at the end of the subjective target interval, a response was positive if the probe was present in the memory set, negative if it was absent. Immediately after the end of the time interval, the subject was asked to report the probe's position in the memory set. Therefore, during the time interval, a decision was made on the presence of the probe in the memory set (processing of item information), and the elements of the memory set had to be maintained in their correct position during the time interval production (maintenance of order information). This condition, memory search + maintenance of order information + time production (MoP), will be compared with an MP condition. If maintenance of order interferes with time estimation, as pro- 
a)

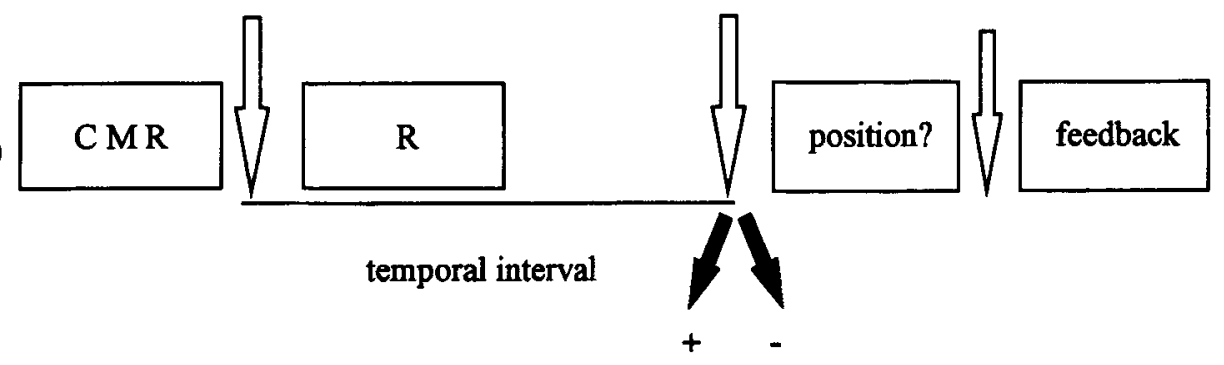

b)

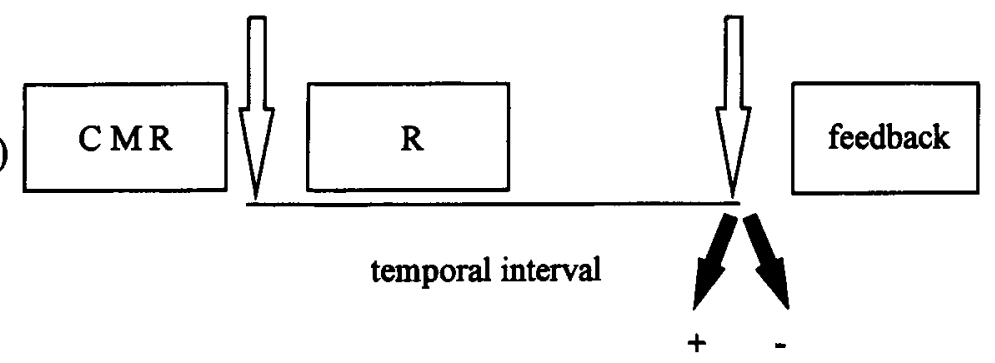

Figure 4. Schematic description of experimental trials in the memory search + maintenance of order information + time production (a) and memory search + time processing (b) conditions in Experiment 2. The main difference with the concurrent processing trials in Experiment 1 is that a question concerning the location of the probe is presented after the end of the time interval production in the MoP condition.

cessing of order did in Experiment 1, an interaction between the memory conditions (MP and MoP) and set size should also be observed in Experiment 2.

\section{Method}

The general method was similar to that of Experiment 1, with some exceptions, described in the following sections.

\section{Subjects}

Forty paid subjects, 17 male and 23 female, between 19 and 36 years old $($ mean age $=27.0)$ took part in this experiment.

\section{Apparatus and Stimuli}

Stimuli were identical to those of Experiment 1, except that five memory set sizes were used $(n=1-5)$ and no stimuli was presented below the probe.

\section{Procedure}

Twenty subjects were randomly assigned to the MP condition, 20 others to the MoP condition. After three temporal production practice sessions, two experimental sessions were completed, comprising one 48-trial block of practice with temporal production alone, including feedback on the temporal performance, and then of four 40-experimental-trial blocks in which temporal production was combined with memory search. In the MP condition, immediately after the end of the temporal production, feedback, indicating whether the response to the search was correct or not, was provided. This was followed by a fixation point, indicating the beginning of the next trial. As is shown in Figure 4, a short question appeared on the screen after the end of the time interval production in the MoP condition: The subject was asked to indicate the position of the probe in the memory set by pressing the corresponding digit (1 to 5 ) on the numerical keyboard, and by pressing the " 0 " key if the probe was not in the memory set. After this response, which was not speeded, the subject was informed whether the response concerning the presence/absence of the probe was correct or not. This feed- back was followed by the presentation of a fixation point, indicating the beginning of the next trial. No feedback was provided on the response concerning the position of the probe.

There were three factors: one between-groups factor, maintenance of order information (order/no-order, two levels), and two within-groups factors, memory set size $(n=1-5$, five levels) and presence/absence of the probe (two levels). In the 20 positive trials of the experimental blocks, in the MoP condition, each possible location of the probe in the memory set was used at each memory set size. To achieve that aim, there were 5 trials with five items in the memory set. To balance with the other memory set sizes, there were 3 trials with three items in the memory set, and 4 trials with each of the remaining memory set sizes-that is, one, two, and four.

Six thousand four hundred observations were collected in the MP condition, 6,240 in the MoP condition, because 1 subject did not complete the second experimental session in this condition. As in Experiment 1, the factors were memory set size and presence/absence of the probe. In the MP and MoP conditions, respectively, $3.8 \%$ and $3.2 \%$ of the data were removed because of errors in the search task, and then $1.0 \%$ and $0.9 \%$ outliers were eliminated. Concerning the location of the probe, $5.7 \%$ of the responses were incorrect. Repeated-measures ANOVAs were carried out on mean RTs and mean temporal intervals, with one within-subjects factor (memory set size, five levels) and one between-subjects factor (MP and $\mathrm{MoP}$ conditions, two levels).

\section{Results and Discussion}

Mean temporal productions as a function of set size in the MP and MoP conditions are shown in Figure 5. Mean intervals lengthened significantly with memory set size $[F(4,152)=34.34, p<.0001]$, confirming again the interfering effect of increasing complexity of a short-term memory task on concurrent time production. The mean temporal intervals were higher in the MoP condition than in the MP condition, but this difference was not signifi- 


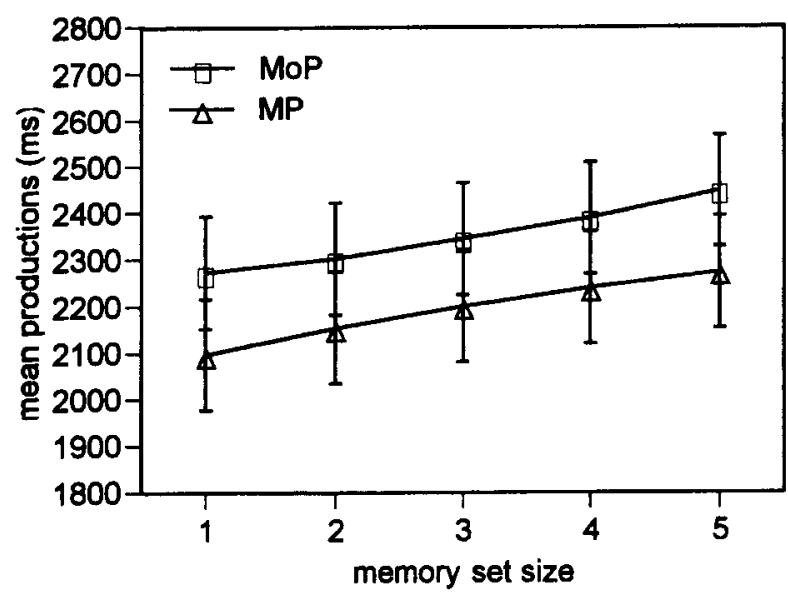

Figure 5. Experiment 2. Mean temporal production (and standard error of the means) as a function of memory set size in the memory search + maintenance of order information + time production (MoP) and memory search + time processing (MP) conditions.

cant $(F<1)$. The interaction between memory set size and order condition was not significant $(F<1)$. If subjects do not need to process order information during the time interval, the effect that was attributed to processing of order in Experiment 1 - namely, a lengthening of productions proportional to set size-disappears. Maintaining order information does not increase the amount of interference proportionally to the number of items in the memory set.

Error rates in the search task - that is, indicating that the probe is present when it is absent or indicating absence when the probe belongs to the memory set-are low: $4.7 \%$ and $3.5 \%$ in the MP and MoP conditions, respectively. The mean percent error rates are shown in Table 2. Mean percent error rates did not differ in the MP and MoP conditions $(F<1)$ but increased significantly with memory set size $[F(4,152)=12.72, p<.0001]$. The interaction between memory set size and order/no-order conditions was also significant $[F(4,152)=3.28, p<.02]$ : error rates tended to increase more rapidly with set size in the MP than in the MoP condition, as can be seen in Table 2. The main result of the analysis of error rates is that errors increased with memory set size.

The results of Experiment 2 suggest that maintaining order information does not interfere with time estimation proportionally to the number of elements to maintain in order. Besides, these results support the interpretation according to which the interaction between processing of order and memory set size observed in Experiment 1 was due to processing of order. Finally, together with the results of Experiment 1, these results suggest that the interference between processing in short-term memory and time estimation depends on the specific operations performed in short-term memory: Whereas processing order information interferes with time estimation, maintenance of order does not have a significant interference effect.

\section{GENERAL DISCUSSION}

In previous studies, various nontemporal tasks of comparable levels of difficulty were combined with time interval production. These experiments showed that the amount of interference between the nontemporal tasks and temporal production was not a function of the level of difficulty of the search task but, instead, of the amount of processing in short-term memory required in the nontemporal task (Fortin \& Rousseau, 1987; Fortin et al., 1993). However, the present study shows that increasing the level of difficulty within a nontemporal task requiring processing in short-term memory increases the interference between the nontemporal task and a concurrent temporal production task: Processing order information, in Experiment 1, increased the slopes of RTs as a function of set size and increased correspondingly the mean temporal productions as a function of set size in a $\mathrm{CP}$ condition. These results support timing models that infer attentional control of a switch (Meck, 1984) or a gate (Rousseau et al., 1984; Zakay \& Block, 1997a) allowing accumulation of temporal information emitted from an internal source. They are also consistent with attentional models of time estimation, in which a negative relation between nontemporal task difficulty and perceived duration of this task has been observed in a prospective time estimation paradigm (see, e.g., Block, 1992; Hicks et al., 1977; Thomas \& Weaver, 1975; Vroon, 1970; Zakay, 1993; Zakay et al., 1983). Furthermore, they are in agreement with conclusions of previous experiments concerning short-term memory control on temporal information accumulation (Fortin et al., 1993). Thus, the amount of interference between a nontemporal task and concurrent time estimation would be specifically related to the level of difficulty of short-term memory processing required in the nontemporal task.

In Experiment 2, the amount of order information maintained in short-term memory did not have a significant effect on concurrent time production. This dissociation between the effects of maintenance and processing in short-term memory on concurrent time production has already been observed in previous experiments with item information: Whereas processing item information lengthens simultaneous temporal intervals proportionally to the number of items to process, varying the number of items to maintain in short-term memory has no effect on simultaneous temporal productions. This was taken as evidence

Table 2

Mean Percent Error Rates for Each Set Size in the Memory Search + Time Processing and Memory Search + Maintenance of Order Information + Time Production Conditions in Experiment 2

\begin{tabular}{lccccc}
\hline & \multicolumn{5}{c}{ Memory Set Size } \\
\cline { 2 - 6 } Condition & 1 & 2 & 3 & 4 & 5 \\
\hline MP & 1.7 & 1.6 & 3.9 & 4.7 & 7.2 \\
MoP & 2.1 & 3.4 & 2.2 & 3.7 & 4.9 \\
\hline
\end{tabular}

Note-MP, memory search + time production; MoP, memory search + maintenance of order + time production. 
that accumulating temporal information, in a time production task, was controlled by processing in short-term or working memory without use of its storage capacity (Fortin \& Breton, 1995). This conclusion is also supported by the results of the present study showing a similar dissociation with order information in short-term memory.

The interference between processing in short-term memory and temporal production has been shown with different types of processing-item recognition, mental rotation of geometrical figures, and rhyme judgments (Fortin \& Breton, 1995; Fortin et al., 1993). The analysis of the interference between order information in shortterm memory and time estimation appears especially interesting because it provides information on the interaction between two types of temporal processing usually studied in relatively distinct lines of research: temporal organization of memory and time estimation (Michon \& Jackson, 1984).

\section{REFERENCES}

BLocK, R. A. (1992). Prospective and retrospective duration judgment: The role of information processing and memory. In F. Macar, V. Pouthas, \& W. J. Friedman (Eds.), Time, action and cognition: Towards bridging the gap (pp. 141-152). Dordrecht: Kluwer.

Chase, W. G. (1977). Does memory scanning involve implicit speech? In S. Dornic (Ed.), Attention and performance VI (pp. 607-628). Hillsdale, NJ: Erlbaum.

Church, R. M. (1984). Properties of the internal clock. In J. Gibbon \& L. Allan (Eds.), Timing and time perception (Annals of the New York Academy of Sciences, Vol. 423, pp. 566-582). New York: New York Academy of Sciences.

FORTIN, C., \& BRETON, R. (1995). Temporal interval production and processing in working memory. Perception \& Psychophysics, 57, 203-215.

Fortin, C., \& Rousseau, R. (1987). Time estimation as an index of processing demand in memory search. Perception \& Psychophysics, 42, 377-382.

Fortin, C., Rousseau, R., Bourque, P., \& Kirouac, E. (1993). Time estimation and concurrent nontemporal processing: Specific interference from short-term-memory demands. Perception \& Psychophysics, 53, 536-548.

Gibbon, J., Church, R. M., \& Meck, W. H. (1984). Scalar timing in memory. In J. Gibbon \& L. Allan (Eds.), Timing and time perception (Annals of the New York Academy of Sciences, Vol. 423, pp. 52-77). New York: New York Academy of Sciences.

Hicks, R. E., Miller, G. W., Gaes, G., \& Bierman, K. (1977). Concurrent processing demands and the experience of time-in-passing. American Journal of Psychology, 90, 431-446.

Hicks, R. E., Miller, G. W., \& Kinsbourne, M. (1976). Prospective and retrospective judgements of time as a function of amount of information processed. American Journal of Psychology, 89, 719-730.
KIRK, R. E. (1995). Experimental design: Procedures for the behavioral sciences ( 3 rd ed.). Belmont, CA: Brooks/Cole.

MaCAR, F., Grondin, S., \& CASINI, L. (1994). Controlled attention sharing influences time estimation. Memory \& Cognition, 22, 673-686.

MCCLAIN, L. (1983). Interval estimation: Effect of processing demands on prospective and retrospective reports. Perception \& Psychophysics, 34, 185-189.

Meck, W. H. (1984). Attentional bias between modalities: Effect on the internal clock, memory, and decision stages used in animal time discrimination. In J. Gibbon \& L. Allan (Eds.), Timing and time perception (Annals of the New York Academy of Sciences, Vol. 423, pp. 528-541). New York: New York Academy of Sciences.

MiCHON, J. A., \& JACKSON, J. L. (1984). Attentional effort and cognitive strategies in the processing of temporal information. In J. Gibbon \& L. Allan (Eds.), Timing and time perception (Annals of the New York Academy of Sciences, Vol. 423, pp. 298-321). New York: New York Academy of Sciences.

Navon, D. (1984). Resources: A theoretical soup stone? Psychological Review, 91, 216-234.

Rousseau, R., PiCARD, D., \& Pitre, E. (1984). An adaptive counter model for time estimation. In J. Gibbon \& L. Allan (Eds.), Timing and time perception (Annals of the New York Academy of Sciences, Vol. 423, pp. 639-642). New York: New York Academy of Sciences.

STERNBERG, S. (1966). High-speed scanning in human memory. Science, 153, 652-654.

STERNBERG, S. (1967). Retrieval of contextual information from memory. Psychonomic Science, 8, 55-56.

STERNBERG, S. (1969). Memory scanning: Mental processes revealed by reaction time experiments. American Scientist, 57, 421-457.

Thomas, E. A. C., \& Weaver, W. B. (1975). Cognitive processing and time perception. Perception \& Psychophysics, 17, 363-367.

TownSEND, J. T., \& AsHBY, F. G. (1983). Stochastic modeling of elementary psychological processes. Cambridge: Cambridge University Press.

VROON, P. A. (1970). Effects of presented and processed information on duration experience. Acta Psychologica, 34, 115-121.

WEARden, J. H., \& Ferrara, A. (1993). Subjective shortening in humans' memory for stimulus duration. Quarterly Journal of Experimental Psychology, 46B, 163-186.

ZAKAY, D. (1990). The evasive art of subjective time measurement: Some methodological dilemmas. In R. A. Block (Ed.), Cognitive models of psychological time (pp. 59-84). Hillsdale, NJ: Erlbaum.

ZAKAY, D. (1993). Time estimation methods-do they influence prospective duration estimates? Perception, 22, 91-101.

ZAKAY, D., \& BLOCK, R. A. (1997a). The role of attention in time estimation processes. In M. A. Pastor \& J. Artieda (Eds.), Time, internal clocks and movement (pp. 143-164). Amsterdam: Elsevier.

ZaKaY, D., \& Block, R. A. (1997b). Temporal cognition. Current Directions in Psychological Science, 6, 12-16.

Zakay, D., Nitzan, D., \& Glickson, J. (1983). The influence of task difficulty and external tempo on subjective time estimation. Perception \& Psychophysics, 34, 451-456.

(Manuscript received May 28, 1997; revision accepted for publication February 6, 1998.) 